\title{
Dynamic Tensor Recommender System
}

\author{
Annie Qu \\ University of Illinois at Urbana-Champaign, USA \\ anniequ@illinois.edu
}

\begin{abstract}
In this talk, we propose a new dynamic tensor recommender system, which aims particularly at long-term recommendation. The proposed method utilizes a tensor-valued function of time to integrate time information and contextual information, and creates a time-varying coefficient canonical polyadic decomposition with grouping effects through polynomial spline approximation. Major advantages are that the proposed method is able to provide competitive long-term recommendations and prediction intervals. Specifically, it provides smooth extrapolation through penalized polynomial splines effectively. In theory, we establish asymptotic consistency of the estimated parameter and asymptotic normality of the estimated coefficients. The proposed method is applied to simulations and IRI marketing data. Numerical studies demonstrate that the proposed method outperforms existing methods in long-term forecasting.
\end{abstract}

